\title{
Bulk viscous corrections to screening and damping in the deconfined phase at high temperature
}

\author{
Adrian Dumitru*i \\ Department of Natural Sciences, Baruch College, CUNY, 17 Lexington Avenue, New York, NY \\ 10010, USA \\ The Graduate School and University Center, The City University of New York, 365 Fifth Avenue, \\ New York, NY 10016, USA \\ Physics Department, Brookhaven National Lab, Upton, NY 11973, USA \\ E-mail: adrian.dumitru@baruch. cuny • edu
}

\begin{abstract}
Non-equilibrium corrections in a hot QCD medium modify the "hard thermal loops" (HTL) which determine the resummed propagators for gluons with soft momenta as well as the Debye screening and Landau damping mass scales. We focus on bulk viscous corrections to a thermal fixed point. The screening and damping mass scales are sensitive to the bulk pressure and perhaps to (pseudo) critical dynamical scaling of the bulk viscosity in the vicinity of a second-order critical point. This would affect the properties of quarkonium bound states in the deconfined phase.
\end{abstract}

Critical Point and Onset of Deconfinement - CPOD2017

7-11 August, 2017

The Wang Center, Stony Brook University, Stony Brook, NY

\footnotetext{
${ }^{*}$ Speaker.

${ }^{\dagger}$ I thank CPOD 2017 for the opportunity to present this work and Qianqian Du, Yun Guo, and Michael Strickland for their collaboration on the material presented in secs. 2 and 3. I also thank Ho-Ung Yee and S. Mukherjee for useful comments at the meeting. I am grateful for support by the DOE Office of Nuclear Physics through Grant No. DE-FG0209ER41620; and from The City University of New York through the PSC-CUNY Research grant 60262-0048.
} 


\section{Introduction, bulk viscosity in QCD}

The bulk viscosity $\zeta(T)$ in QCD at very high temperatures $T \gg \Lambda_{\mathrm{QCD}}$ has been computed to leading order in the coupling in Ref. [1]. They find that it is very small since $\zeta$ is proportional to the square of the deviation from conformality given by the $\beta$-function. This leads to $\zeta / \eta \sim \alpha_{s}^{4}$ (neglecting logarithms of the inverse coupling) which is proportional to

$$
\left(\alpha_{s}^{2} N^{2}\right)^{2} \sim\left(1-3 c_{s}^{2}\right)^{2}
$$

$c_{s}$ is the speed of sound. To see this write the trace anomaly in the pure glue theory in the form [2]

$$
\beta\left(\alpha_{R}\right) \frac{\partial}{\partial \alpha_{R}} p\left(T, \alpha_{R}\right)=e-3 p .
$$

Here, $\alpha_{R}$ denotes the renormalized coupling at a scale $\mu$ and $p\left(T, \alpha_{R}\right)$ is the pressure as a function of $T$ and $\alpha_{R}$. On the 1.h.s. the derivative w.r.t. the renormalized coupling is performed at constant $T$. In (pure glue) perturbation theory, up to first order in the running coupling $\alpha_{s}\left(T ; \alpha_{R}\right)$ :

$$
p\left(T, \alpha_{R}\right)-p(T, 0)=-\frac{N\left(N^{2}-1\right)}{144} \alpha_{s}(T) T^{4}
$$

and eq. (1.2) turns into

$$
\begin{aligned}
e-3 p & =-\frac{N\left(N^{2}-1\right)}{144} T^{4} \beta\left(\alpha_{R}\right) \frac{\partial}{\partial \alpha_{R}} \alpha_{s}(T), \\
& =-\frac{N\left(N^{2}-1\right)}{144} T^{4} \beta\left(\alpha_{s}(T)\right) .
\end{aligned}
$$

Differentiate both sides w.r.t. the energy density; on the r.h.s. write this derivative as $(\partial e / \partial T)^{-1} \partial / \partial T$ and note that to leading order this latter derivative w.r.t. $T$ acts on the factor $T^{4}$ only:

$$
1-3 c_{s}^{2}=-\frac{\frac{N\left(N^{2}-1\right)}{36} T^{3} \beta\left(\alpha_{s}(T)\right)}{\partial e / \partial T} .
$$

Thus, $1-3 c_{s}^{2}$ is indeed proportional to $-N \beta\left(\alpha_{s}(T)\right) \sim \alpha_{s}^{2}(T) N^{2}$.

On the other hand, when $(e-3 p) / T^{4}$ exhibits a power law tail then the proportionality (1.1) can be modified. For illustration consider a simple model where a non-perturbative contribution [3] is added to the one-loop pressure while the two-loop pressure remains ${ }^{1}$ the one from eq. (1.3):

$$
p(T)=\left(N^{2}-1\right) \frac{\pi^{2}}{45}\left(T^{4}-T^{2} T_{c}^{2}\right)-\frac{N\left(N^{2}-1\right)}{144} \alpha_{s}(T) T^{4} .
$$

At order $\alpha_{s}^{0}$ this gives

$$
\begin{aligned}
e-3 p & =2\left(N^{2}-1\right) \frac{\pi^{2}}{45} T^{2} T_{c}^{2} \approx 0.44\left(N^{2}-1\right) T^{2} T_{c}^{2}, \\
c_{s}^{2} & =\frac{2 T^{2}-T_{c}^{2}}{6 T^{2}-T_{c}^{2}} .
\end{aligned}
$$

\footnotetext{
${ }^{1}$ The assumption that the two-loop pressure is unaffected by the non-perturbative contribution does not correspond to the models proposed in refs. [3].
} 
$(e-3 p) / T^{2} T_{c}^{2}$ in fact agrees quite well with (pure gauge) lattice data [4, 5] for $N=3,4,6$ colors in the regime $T / T_{c} \simeq 1.5-4$. One can now obtain the running coupling $\alpha_{S}(T)$ from the trace anomaly. To do so, replace the 1.h.s. of eq. (1.4) by (1.7):

$$
N \beta\left(\alpha_{s}(T)\right)=-\frac{288 \pi^{2}}{45} \frac{T_{c}^{2}}{T^{2}} \quad \rightarrow \quad N \alpha_{s}(T)=\frac{144 \pi^{2}}{45} \frac{T_{c}^{2}}{T^{2}} \quad, \quad \beta\left(\alpha_{s}\right)=-2 \alpha_{s} .
$$

This result for the running coupling shows explicitly why this illustration is not self consistent: in eq. (1.6), the contribution to the pressure at order $\alpha_{s}$ is comparable to the leading contribution, just like the pure perturbative expansion of the pressure. Nevertheless, to complete this toy model calculation use eq. (1.8) to express $1-3 c_{s}^{2}$ in terms of $\alpha_{s}(T)$ :

$$
1-3 c_{s}^{2}(T)=\frac{90 N \alpha_{s}(T)}{864-45 N \alpha_{s}(T)} .
$$

Hence, at $T / T_{c}=4$, say, where $\alpha_{s} N$ is sufficiently small, $1-3 c_{s}^{2}$ would be approximately linear instead of quadratic in $\alpha_{s} N$ (and linear in $-\beta N$ ).

In a more realistic theory of the deconfined phase one should not expect that $1-3 c_{s}^{2}(T)$ is given simply by a number times a power of $\alpha_{s}(T)$. Rather, that "number" presumably would be a dimensionless function $f\left(T / T_{c}, T / m_{D}, \ldots\right)$ of temperature. However, if that theory does not involve or generate $T_{c}$ then a fit of the form $1-3 c_{s}^{2}(T)=$ const. $\times\left(\alpha_{s}(T) N\right)^{b(T)}$ would still be interesting. In particular, a fit to $N_{f}=2+1$ three loop HTLpt over $T=0.15 \rightarrow 1.5 \mathrm{GeV}$ (at $\mu_{B}=0$ ) with constant power $b$ gives $b \approx 3.3$ [6].

For completeness we mention also that in the limit of $N \rightarrow \infty$ and large 't Hooft coupling $\lambda \gg 1$ the holographic correspondence for broken conformal invariance suggests $\zeta / \eta \sim 1-3 c_{s}^{2}$ [7] in 3 spatial dimensions; also see refs [8].

For heavy-ion collisions the most relevant temperature regime is $T \lesssim 4 T_{c}$. The lattice has shown that the trace anomaly of QCD, expressed as energy density minus three times the pressure, grows large at $T \sim T_{c}$ [9]. Thus, it has been suggested in the literature that the bulk viscosity to entropy density ratio should increase, too, as the temperature approaches the confinementdeconfinement temperature [10]. Here we perform a weak-coupling HTL computation to assess, at least qualitatively, the impact of bulk-viscous corrections on the heavy-quark potential. This analysis does not apply for $T \simeq T_{c}$ but it provides baseline expectations for bulk-viscous effects on screening and damping from (resummed) weakly coupled QCD.

Bulk viscous corrections are expected to become important also in the vicinity of a second order critical point; this could be realized in hot QCD either by tuning of the quark masses [11] or perhaps by introducing a baryon charge asymmetry [12]. Due to critical slowing down the bulk viscosity should diverge [13] $\zeta \sim \xi^{z}$ where $\xi \rightarrow \infty$ is the correlation length and $z$ is a dynamical critical exponent. However, since the relaxation time in the critical region of the bulk pressure diverges as well, in heavy-ion collisions it is not expected to exceed the ideal pressure [14].

\section{HTL resummed gluon self energies incl. bulk-viscous corrections}

To compute the gluon self energy in the hard loop approximation we require the phase space distributions of the particles in the medium. In the local rest frame, we take them to be

$$
f(\mathbf{p})=f_{\mathrm{id}}(p)+\delta_{\text {bulk }} f(p)+\delta_{\text {shear }} f(\mathbf{p}) .
$$


Here, $f_{\text {id }}(p)$ is an isotropic reference distribution when non-equilibrium corrections are absent. This would normally correspond to thermal Fermi-Dirac or Bose-Einstein distributions, respectively, if the "ideal" reference is the thermal fixed point.

The corrections $\delta f$ in Eq. (2.1) correspond to non-equilibrium corrections. We take the isotropic correction $\delta_{\text {bulk }} f(p)$ as a bulk-viscous correction while the anisotropic part $\delta_{\text {shear }} f(\mathbf{p})$ is analogous to shear. However, we do not assume that these corrections are parametrically suppressed. The corrections to the real and imaginary parts of the HTL resummed gluon propagator due to $\delta_{\text {shear }} f(\mathbf{p})$ have been worked out in Refs. [15]. Here, we focus on bulk viscous corrections instead.

To obtain explicit results for the self energies we require a model for $\delta_{\text {bulk }} f(k)$. We assume that the bulk viscous correction to the local thermal distribution function takes the form

$$
\delta_{\text {bulk }} f(k)=\left(\frac{k}{T}\right)^{a} \Phi f_{\text {id }}(k)\left(1 \pm f_{\text {id }}(k)\right) .
$$

$\Phi$ is proportional to the bulk pressure, which in the Navier-Stokes / Landau approximation is negative, and $a$ is a constant. We require $a>0$ to ensure that the dominant contribution to the retarded self energy is from hard (gluon) loop momenta, $k \sim T$. The bulk viscous correction to the symmetric self energy at $\mathscr{O}\left(\Phi^{2}\right)$ involves the fourth power of the distribution function and requires a more stringent bound, $a>1 / 2$. It should be clear that (2.2) is a generic (and simple) model for the non-equilibrium correction chosen such as to maintain applicability of HTL power counting which may in principle be violated in certain non-equilibrium scenarios. Furthermore, we recall that if $\delta_{\text {bulk }} f(k)$ does not vanish then the hard scale $T$ in the distributions functions is no longer equal to the temperature which instead must be determined from the Landau matching condition. However, the hard scale remains on the order of the temperature and we continue to denote it as $T$ for simplicity of presentation. Finally, we shall further assume that $|\Phi| \gg g^{2}$ so that two-loop corrections to the gluon self energy are negligible.

Using standard methods of real-time thermal field theory [16] one can derive [17] the following expressions for the retarded and symmetric time-ordered (temporal) gluon self energies ${ }^{2}$ :

$$
\begin{aligned}
& \Pi_{R}(P)=\frac{N_{f} g^{2}}{(2 \pi)^{2}} \int k d k f_{F}(k) \int d \Omega_{k} \frac{1-(\hat{\mathbf{k}} \cdot \hat{\mathbf{p}})^{2}}{\left(\hat{\mathbf{k}} \cdot \hat{\mathbf{p}}+\frac{p_{0}+i \varepsilon}{p}\right)^{2}}, \\
& \Pi_{F}(P)=-i \frac{N_{f} g^{2}}{\pi} \frac{2}{p} \Theta\left(p^{2}-p_{0}^{2}\right) \int k^{2} d k f_{F}(k)\left(1-f_{F}(k)\right) .
\end{aligned}
$$

The advanced self energy is equal to $\Pi_{R}(P)$ with an inverted sign of $\varepsilon$. These expressions account for the contribution due to $N_{f}$ loops of massless quarks. For the contribution due to a gluon loop replace the Fermi-Dirac distribution $f_{F}(k)$ by a Bose distribution $f_{B}(k)$, Pauli blocking $1-f_{F}(k)$ by Bose enhancement $1+f_{B}(k)$, and $N_{f}$ by $N_{c}$. Here, $f(k)$ corresponds to the non-equilibrium distribution function introduced in eqs. $(2.1,2.2)$ above.

It is clear from eqs. $(2.3,2.4)$ that for isotropic $\delta f$ the dependence of the self energies on the external energy/momentum is the same as in equilibrium. However, there is a correction to the

\footnotetext{
${ }^{2}$ For brevity we restrict to vanishing quark chemical potential, see ref. [17] for the corresponding expressions at $\mu>0$.
} 
mass scales which appear in $\Pi_{R}(P)$ and $\Pi_{F}(P)$ :

$$
\begin{aligned}
m_{R}^{2}=\left(2 N_{c}+N_{f}\right) \frac{g^{2} T^{2}}{6} \rightarrow & m_{R}^{2}+\delta m_{R}^{2}= \\
& \left(2 N_{c}\left(1+c_{R}^{(g)}(a) \Phi\right)+N_{f}\left(1+c_{R}^{(q)}(a) \Phi\right)\right) \frac{g^{2} T^{2}}{6}, \\
m_{F}^{2}=\left(2 N_{c}+N_{f}\right) \frac{g^{2} T^{2}}{6} \rightarrow & m_{F}^{2}+\delta m_{F}^{2}= \\
& \left(2 N_{c}\left(1+c_{F}^{(g)}(a) \Phi\right)+N_{f}\left(1+c_{F}^{(q)}(a) \Phi\right)\right) \frac{g^{2} T^{2}}{6} .
\end{aligned}
$$

In (2.6) only the correction linear in $\Phi$ has been given, see ref. [17] for $m_{F}^{2}+\delta m_{F}^{2}$ to order $\Phi^{2}$. Here,

$$
\begin{aligned}
c_{R}^{(a)}(a) & =2\left(1-2^{-a}\right) c_{R}^{(g)}(a)=\frac{12}{\pi^{2}}\left(1-2^{-a}\right) \Gamma(2+a) \zeta(1+a), \\
c_{F}^{(q)}(a) & =2\left(1-2^{-a}\right) c_{F}^{(g)}(a)=\frac{6}{\pi^{2}}\left(1-2^{-a}\right) \Gamma(3+a) \zeta(1+a),
\end{aligned}
$$

are pure numbers of order 1. Therefore, a substantial (negative) bulk-viscous pressure $\Phi$ could potentially "short out" the self-energies. It would be interesting to check the values of $m_{R}^{2}$ and $m_{F}^{2}$ obtained in a hydrodynamic simulation which incorporates the critical behavior of the bulk viscosity (such as presented in ref. [14]). Non-equilibrium effects on the gluon self energies (or the heavy-quark potential) should also be visible in transport theory approaches [18].

The Schwinger-Dyson equation determines the HTL resummed propagators (in Coulomb gauge),

$$
\begin{aligned}
& D^{*}{ }_{R}(P)=\frac{1}{p^{2}-\Pi_{R}(P)}=\frac{1}{p^{2}-\left(m_{R, D}^{2}+\delta m_{R, D}^{2}\right)\left(\frac{p_{0}}{2 p} \ln \frac{p_{0}+p+i \varepsilon}{p_{0}-p+i \varepsilon}-1\right)}, \\
& D^{*}{ }_{F}(P)=D_{R}^{*}(P) \Pi_{F}(P) D_{A}^{*}(P) .
\end{aligned}
$$

The advanced propagator is equal to $D^{*}{ }_{R}(P)$ with a reversed sign of $\varepsilon$.

\section{Non-equilibrium corrections to the HTL static potential}

We can compute the static potential due to one gluon exchange through the Fourier transform of the physical "11" Schwinger-Keldysh component of the (longitudinal) gluon propagator in the static limit,

$$
\begin{aligned}
V(\mathbf{r}) & =(i g)^{2} C_{F} \int \frac{d^{3} \mathbf{p}}{(2 \pi)^{3}}\left(e^{i \mathbf{p} \cdot \mathbf{r}}-1\right)\left(D^{*}\left(p_{0}=0, \mathbf{p}\right)\right)_{11} \\
& =-g^{2} C_{F} \int \frac{d^{3} \mathbf{p}}{(2 \pi)^{3}}\left(e^{i \mathbf{p} \cdot \mathbf{r}}-1\right) \frac{1}{2}\left(D^{*}{ }_{R}+D^{*}{ }_{A}+D^{*}{ }_{F}\right) .
\end{aligned}
$$

We have also subtracted an $r$-independent (but $T$-dependent) self-energy contribution. The Fourier transform of the sum of retarded and advanced propagators gives a real Debye screened potential,

$$
\operatorname{Re} V(r)=-\frac{g^{2} C_{F}}{4 \pi r} e^{-\hat{r}}
$$


where $\hat{r} \equiv r \sqrt{m_{R}^{2}+\delta m_{R}^{2}}$. The imaginary part of the potential originates from the symmetric propagator $D^{*}{ }_{F}$ and is due to Landau damping of the gluon exchanged by the static sources [19],

$$
\operatorname{Im} V(r)=-\frac{g^{2} C_{F} T}{4 \pi} \frac{m_{F}^{2}+\delta m_{F}^{2}}{m_{R}^{2}+\delta m_{R}^{2}} \phi(\hat{r})
$$

with $\phi(\hat{r}) \sim \hat{r}^{2} \log 1 / \hat{r}$ when $\hat{r} \ll 1$. The imaginary part of the potential generates a thermal width for quarkonium bound states.

Hence, bulk-viscous corrections in general modify the Debye screening of the $1 / r$ Coulomb potential. Also, $\operatorname{Im} V(r)$ is multiplied by $\left(m_{F}^{2}+\delta m_{F}^{2}\right) /\left(m_{R}^{2}+\delta m_{R}^{2}\right)$ which we expect to be less than 1 typically. Thus, a significant bulk pressure should reduce the thermal width of quarkonium states. The potential written in eqs. $(3.2,3.3)$ is not expected to provide accurate, quantitative predictions of the properties of quarkonium bound states at finite temperature. However, basic qualitative insight obtained with the methods described here can be useful for incorporating non-equilibrium effects into quarkonium potentials derived from lattice QCD [20].

\section{References}

[1] P. B. Arnold, C. Dogan and G. D. Moore, Phys. Rev. D 74, 085021 (2006).

[2] I. T. Drummond, R. R. Horgan, P. V. Landshoff and A. Rebhan, Phys. Lett. B 460, 197 (1999)

[3] P. N. Meisinger, T. R. Miller and M. C. Ogilvie, Phys. Rev. D 65, 034009 (2002);

R. D. Pisarski, Phys. Rev. D 74, 121703 (2006);

A. Dumitru, Y. Guo, Y. Hidaka, C. P. Korthals Altes and R. D. Pisarski, Phys. Rev. D 86, 105017 (2012)

[4] T. Umeda, S. Ejiri, S. Aoki, T. Hatsuda, K. Kanaya, Y. Maezawa and H. Ohno, Phys. Rev. D 79, 051501 (2009);

S. Borsanyi, G. Endrodi, Z. Fodor, S. D. Katz and K. K. Szabo, JHEP 1207, 056 (2012).

[5] M. Panero, Phys. Rev. Lett. 103, 232001 (2009);

S. Datta and S. Gupta, Phys. Rev. D 82, 114505 (2010)

[6] J. O. Andersen, L. E. Leganger, M. Strickland and N. Su, Phys. Rev. D 84, 087703 (2011); JHEP 1108, 053 (2011)

and M. Strickland, priv. comm.

[7] A. Buchel, Phys. Lett. B 663, 286 (2008)

[8] S. S. Gubser, S. S. Pufu and F. D. Rocha, JHEP 0808, 085 (2008);

C. Eling and Y. Oz, JHEP 1106, 007 (2011);

S. I. Finazzo, R. Rougemont, H. Marrochio and J. Noronha, JHEP 1502, 051 (2015)

[9] A. Bazavov et al. [HotQCD Collaboration], Phys. Rev. D 90, 094503 (2014).

[10] D. Kharzeev and K. Tuchin, JHEP 0809, 093 (2008);

F. Karsch, D. Kharzeev and K. Tuchin, Phys. Lett. B 663, 217 (2008).

[11] F. R. Brown, F. P. Butler, H. Chen, N. H. Christ, Z. h. Dong, W. Schaffer, L. I. Unger and A. Vaccarino, Phys. Rev. Lett. 65, 2491 (1990);

S. Gavin, A. Gocksch and R. D. Pisarski, Phys. Rev. D 49, R3079 (1994). 
[12] A. M. Halasz, A. D. Jackson, R. E. Shrock, M. A. Stephanov and J. J. M. Verbaarschot, Phys. Rev. D 58, 096007 (1998);

M. A. Stephanov, K. Rajagopal and E. V. Shuryak, Phys. Rev. Lett. 81, 4816 (1998);

J. Berges and K. Rajagopal, Nucl. Phys. B 538, 215 (1999)

[13] G. D. Moore and O. Saremi, JHEP 0809, 015 (2008).

[14] A. Monnai, S. Mukherjee and Y. Yin, Phys. Rev. C 95, no. 3, 034902 (2017)

[15] A. Dumitru, Y. Guo and M. Strickland, Phys. Lett. B 662, 37 (2008); Phys. Rev. D 79, 114003 (2009); M. Nopoush, Y. Guo and M. Strickland, JHEP 1709, 063 (2017)

[16] S. Mrowczynski and M. H. Thoma, Phys. Rev. D 62, 036011 (2000);

M. E. Carrington, De-fu Hou and M. H. Thoma, Phys. Rev. D 58, 085025 (1998); Eur. Phys. J. C 7, 347 (1999);

P. B. Arnold, D. T. Son and L. G. Yaffe, Phys. Rev. D 59, 105020 (1999)

[17] Q. Du, A. Dumitru, Y. Guo and M. Strickland, JHEP 1701, 123 (2017)

[18] S. Y. F. Liu and R. Rapp, Nucl. Phys. A 941, 179 (2015)

[19] M. Laine, O. Philipsen, P. Romatschke and M. Tassler, JHEP 0703, 054 (2007);

N. Brambilla, J. Ghiglieri, A. Vairo and P. Petreczky, Phys. Rev. D 78, 014017 (2008);

M. A. Escobedo and J. Soto, Phys. Rev. A 78, 032520 (2008).

[20] B. Krouppa, A. Rothkopf and M. Strickland, arXiv:1710.02319 [hep-ph]. 